\title{
Preoperative risk factors for postoperative delirium (POD) after urological surgery in the elderly
}

\author{
P. Tognoni ${ }^{\text {a }}$, A. Simonato ${ }^{\text {a }}$, N. Robutti ${ }^{\text {b }}$, M. Pisani ${ }^{\text {a }}$, A. Cataldi ${ }^{\text {b }}$, F. Monacelli $^{\text {b }}$, G. Carmignani $^{\text {a }}$, \\ P. Odetti ${ }^{\mathrm{b}, *}$ \\ ${ }^{a}$ Department of Urology, Urology section University-Hospital San Martino, Viale Benedetto XV, n. 6, I-16132 Genoa, Italy

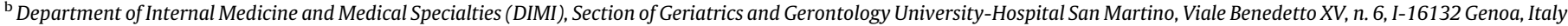

\section{A R T I C L E I N F O}

\section{Article history:}

Received 10 July 2010

Received in revised form 19 October 2010

Accepted 20 October 2010

Available online 16 November 2010

\section{Keywords:}

Delirium

Urological surgery

Older people

Hypotension

Multidimensional assessment

\begin{abstract}
A B S T R A C T
The aim of this observational study was to investigate the occurrence of postoperative delirium (POD) in elderly patients undergoing urological surgery and to identify those factors associated with delirium. Ninety consecutive patients ( 81 males and 9 females; average age of $74.3 \pm 0.40$ years), undergoing urological surgery in University-Hospital Urological Clinic were selected. Personal, medical, cognitive and functional data, biochemical parameters, preoperative medications, conduct of surgery and anesthesia and details of hemodynamic control were collected as predictors of delirium. After surgery, the subjects were divided on the basis of delirium onset within a week observation period. Delirium was diagnosed by the Confusion Assessment Method. Delirium started the first post-operative day (2F; $6 \mathrm{M}$ ) and lasted $3.0 \pm 0.8$ days. Subjects with POD were significantly older, had a previous history of delirium, were more impaired in the instrumental activities of daily living and had poorer clock drawing test (CDT) score. Interestingly, a significantly greater number of hypotensive events were recorded during anesthesia. Age, cognitive and functional status, previous history of delirium and hypotensive episodes intrasurgery are the best predictor of POD in this setting. Our findings have implications in preventing delirium in elderly by an early and targeted evaluation.
\end{abstract}

๑ 2010 Elsevier Ireland Ltd. All rights reserved.

\section{Introduction}

The people over 65 years as a group has increased rapidly in the recent years and demographic projections predict a progressive future increase (United Nations, 2002). Older age is characterized by aging of tissues and organs, a greater occurrence of cognitive decline and functional impairment and an increasing number of frail subjects (Fried et al., 2001; Blaum et al., 2002). These frail elderly are more prone to several complications including delirium, which affects approximately one fourth of the hospitalized older patients and a wide range of post-surgical elderly (Amador and Goodwin, 2005). The elderly are a large group of patients undergoing urological surgery, both for endoscopic and for open surgery and POD is an important issue in the daily clinical practice.

As a consequence of acute confusion (delirium), there is a greater functional decline, an increased morbidity, a longer stay in the acute hospital unit and a higher rate of nursing home placement (Minden et al., 2005). Despite the significant incidence

\footnotetext{
* Corresponding author. Tel.: +39 010353 7985; fax: +39 0103537985 . E-mail address: odetti@unige.it (s.\$. Odetti).
}

rate, delirium has not been often diagnosed by the physicians: perhaps because it is grossly underestimated or due to the presence of confounding factors (Meagher, 2001). Delirium, in elderly patients, is a potential consequence of the surgical approach with a serious impact on the quality of life.

Several studies evaluated various risk factors (Inouye and Charpentier, 1996; Litaker et al., 2001) to identify those most useful in predicting the onset of delirium and the outcome development, since an early recognition of the symptoms or a correction of predisposing factors may prevent delirium or let to initiate a specific treatment. The aim of this study was to investigate the potential risk factors of acute confusion in a group of aged patients undergoing urologic procedures.

\section{Subjects and methods}

\subsection{Patients}

\subsubsection{Inclusion criteria}

Ninety patients, 81 males and 9 females, with an average age of $74.3 \pm 0.40$ years (range 66-93 years), were recruited for the study. The admission for disease with severe functional or cognitive impairment (mini mental state examination $=$ MMSE $<10$ ) was used 
as exclusion criteria. Inclusion criteria were major (open) and minor (endoscopic) surgery. Regarding open surgery we included patients undergoing transvescical prostatectomy (TVP), radical retropubic prostatectomy (RRP), radical nephrectomy (RN), radical cystectomy (RC).

Regarding minor-endoscopic surgery, we elected to include patients undergoing trans-urethral resection of prostate (TURP) that exceeded $60 \mathrm{~min}$ of surgical session. Individuals with superficial bladder cancers, which can be small and treatable with a very short surgical session, were not included in the study.

A complete description of the research protocol was provided to all patients, and then a written informed consent to participate to this study was obtained.

\subsection{Personal data}

Medical and social data were collected from the patients and their caregivers when needed. Pharmacological treatment on admission was recorded, in particular the use of benzodiazepines, neuroleptics or drugs with anticholinergic effects.

In all recruited patients previous neurological or psychologicalpsychiatric disorders, previous episodes of delirium and alcohol abuse were collected. Routine preoperative blood test was performed including a complete blood count, a serum electrolyte panel, a liver function test, a urinalysis; thyroid hormones and glycated hemoglobin analyses were performed. Chest radiogram, electrocardiogram (ECG) were also collected.

\subsection{Type of anesthesia}

All open surgeries were conducted using general anesthesia including rapid induction with phentanyl, propofol and succinylcholine followed by orotracheal intubation; maintenance was obtained with sevoflurane mixed with $50 \%$ nitrogen and oxygen, curare and remiphentanyl.

General or regional anesthesias were administered also for endoscopic surgery. General anesthesia was similar to that used for open surgery: fast induction with phentanyl and propofol but followed by placement of a laryngeal masque; maintenance was obtained both with sevoflurane mixed with $50 \%$ nitrogen and oxygen or with propofol in continuous intravenous perfusion. Regional spinal anesthesia was performed injecting $1.6-2 \mathrm{ml}$ of bupivacaine $1 \%$ in the L2-L3 or L3-L4 vertebral space. The choice for general or regional anesthesia was based on the clinical condition and/or preference of the individual patient.

Intra-operative data from the anesthesia record were also reviewed. Systolic and diastolic blood pressure were monitored every 15 min during the operation and any systolic blood pressure drop in relation to the blood pressure before administration of anesthesia were recorded in percentages.

Hypotension was defined as a systolic blood pressure decline to $<90 \mathrm{mmHg}$ requiring pressors or fluids resuscitation. Duration of hypotension was also recorded. Particular attention was taken to avoid low oxygen saturation or pain in the postoperative period. Oxygen was administered at regular intervals according to the clinical request; pain was generally controlled with a lumbar epidural catheter or with intravenous patient-controlled analgesia (PCA) for the main open procedures.

Based on clinical results, these supports were stopped as soon as possible, and a non-steroidal anti-inflammatory drug was started in the follow-up.

\subsection{Neuropsychological and multidimensional assessment}

Preoperatively, a trained research assistant administered a battery for mental assessment, as MMSE, the Yesavage's geriatric depression scale (GDS) short version, CDT using the Mendez calculation score (Mendez et al., 1992), and functional assessment, as the activities of daily living (ADL) and instrumental activities of daily living (IADL). All the patients were evaluated postoperatively, daily, for a week and at the onset of confusion related symptoms, the confusion assessment method (CAM) was performed, according to the DSM-IV TR (2000). CAM was utilized because is more easily explained and interpreted than other scales; according to it, delirium was considered when 4 basic criteria were satisfied: acute and fluctuating starting, lowering of attention, thought disorganization, alteration of alertness.

\subsection{Statistical calculations}

All data are expressed as mean \pm standard error of mean (SEM). Unpaired Student's $t$ test was used for the comparison between two groups. When appropriate, univariate analysis was performed utilizing the Fisher's exact test. The statistical tests were performed using the Prism software package (vers. 4.02 for Mac-GraphPad Software, San Diego, California, USA). A probability lower than $5 \%$ $(p<0.05)$ was considered statistically significant.

\section{Results}

Fifty-six patients underwent open surgery and 34 endoscopic procedures; general anesthesia was employed in 68 patients while 22 had spinal anesthesia. Two females and six males of the 90 recruited patients (8.8\%), developed POD. The various surgical procedures and anesthetics used for all patients and those experiencing POD are listed in Table 1.

The onset of transient delirium occurred on the first postoperative day and lasted $3.0 \pm 0.8$ days. The characteristics of urological surgery patients with or without transient delirium are shown in Table 2.

One or more comorbidity conditions were more frequent $(62.5 \%)$ in the delirious patients than in the non-delirious patients (55\%), but the difference was not statistically significant (n.s.). No differences were seen in delirious $(1.75 \pm 0.56$ medications) compared to non-delirious patients $(2.12 \pm 0.23$ medications $)$ in the drugs prescribed in both groups before admission to the hospital (Table 2).

There was no difference between patients with or without delirium and the prevalence of open surgery or the use of general anesthesia (Table 2). The information contained in Table 2 show that patients with POD were significantly older, had greater personal history of delirium, had a poorer CDT score and were more impaired in the IADL.

Among the baseline parameters delirious patients had similar values of biochemical and haematological values as well as for thyroid function testing. A significantly higher incidence of hypotension was recorded in delirious patients than in nondelirious ones ( $5 / 8$ vs. 20/82; $p<0.05$ ) (Table 2). As expected,

\section{Table 1}

Surgical procedures and types of anaesthesia in our study group (number of delirious patients are in the parentheses).

\begin{tabular}{llll}
\hline Surgery type & \multicolumn{2}{l}{ Types of anaesthesia } & Total \\
\cline { 2 - 3 } & Regional & General & \\
\hline TURP & $22(3)$ & 12 & $34(3)$ \\
TVP & & $18(2)$ & $18(2)$ \\
RRP & 18 & 18 \\
RN & & $13(1)$ & $13(1)$ \\
RC & $7(2)$ & $7(2)$ \\
Total & $22(3)$ & $68(5)$ & $90(8)$ \\
\hline
\end{tabular}


Table 2

Preoperative clinical, mental and functional characteristics of patients undergone urologic surgery. Comparison between POD vs. not delirious patients.

\begin{tabular}{|c|c|c|c|c|c|c|}
\hline \multirow[t]{2}{*}{ Number } & & \multicolumn{2}{|c|}{$\begin{array}{l}\text { No delirium } \\
82\end{array}$} & \multicolumn{2}{|c|}{$\begin{array}{l}\text { With delirium } \\
8\end{array}$} & \multirow[b]{2}{*}{$\chi^{2}$} \\
\hline & & \multicolumn{2}{|c|}{$n(\%)$} & \multicolumn{2}{|l|}{$n(\%)$} & \\
\hline \multicolumn{2}{|l|}{ Women } & \multicolumn{2}{|c|}{7 (9) } & \multicolumn{2}{|c|}{$2(25)$} & ns \\
\hline \multicolumn{2}{|l|}{ Comorbidity $\geq 2$ diseases } & \multicolumn{2}{|c|}{$45(55)$} & \multicolumn{2}{|c|}{$5(62.5)$} & ns \\
\hline \multicolumn{2}{|l|}{ Previous delirium } & \multicolumn{2}{|c|}{$5(6)$} & \multicolumn{2}{|c|}{$3(37.5)$} & $p=0.003$ \\
\hline \multicolumn{2}{|l|}{ Alcohol consumption } & \multicolumn{2}{|c|}{$7(8.5)$} & \multicolumn{2}{|c|}{$0(0)$} & ns \\
\hline \multicolumn{2}{|c|}{ Open/endoscopic urologic surgery } & \multicolumn{2}{|c|}{$50 / 32$} & \multicolumn{2}{|l|}{$6 / 2$} & ns \\
\hline \multirow{2}{*}{\multicolumn{2}{|c|}{$\begin{array}{l}\text { General/spinal anesthesia } \\
\text { Hypotension during surgery }\end{array}$}} & \multirow{2}{*}{\multicolumn{2}{|c|}{$\begin{array}{l}62 / 20 \\
20(24.4)\end{array}$}} & \multirow{2}{*}{\multicolumn{2}{|c|}{$\begin{array}{l}5 / 3 \\
5(62.5)\end{array}$}} & \multirow{2}{*}{$\begin{array}{l}\text { ns } \\
p=0.022\end{array}$} \\
\hline & & & & & & \\
\hline & \multicolumn{2}{|c|}{ Mean \pm SD } & \multicolumn{2}{|c|}{ Mean \pm SD } & $t=$ & $p=$ \\
\hline Age (years) & \multicolumn{2}{|c|}{$74 \pm 0.4$} & \multicolumn{2}{|c|}{$77 \pm 1.7$} & 2.13 & 0.036 \\
\hline Education (years) & \multicolumn{2}{|c|}{$9.05 \pm 0.44$} & \multicolumn{2}{|c|}{$7.75 \pm 1.71$} & 0.86 & ns \\
\hline No of prescribed drugs & \multicolumn{2}{|c|}{$2.12 \pm 0.23$} & 1.75 & \pm 0.56 & 0.55 & ns \\
\hline MMSE (score) & $27.20 \pm$ & 0.26 & 26.41 & \pm 1.30 & 0.82 & ns \\
\hline CDT (score) & $18.37 \pm$ & 0.29 & 16.25 & \pm 1.42 & 2.05 & 0.040 \\
\hline GDS (score) & $2.46 \pm$ & 0.24 & 3.13 & \pm 0.77 & 0.41 & ns \\
\hline ADL (functions lost) & $0.42 \pm$ & 0.07 & 0.50 & \pm 0.19 & 0.71 & ns \\
\hline IADL (functions lost) & $0.18 \pm$ & 0.08 & 0.88 & \pm 0.39 & 2.37 & 0.017 \\
\hline
\end{tabular}

hypotension was more frequent during open than endoscopic surgery ( $40 \%$ vs. $9 \%, p<0.001)$.

\section{Discussion}

Delirium occurs in hospitalized patients (Inouye and Charpentier, 1996; Inouye, 1998; McCusker et al., 2002; Minden et al., 2005), especially in patients having surgical procedures (Marcantonio et al., 1998; Amador and Goodwin, 2005). The rate of POD is variable depending on the type of surgical and anaesthetic procedures (Papaioannou et al., 2005). Orthopedic surgery has a greater POD (40-60\%), and ophthalmic surgery much lower $(<5 \%)$.

In our group of urological patients, POD occurred in $<10 \%$, confirming the hypothesis that urological surgery is associated with low rate of delirium. However, some interesting results came from the analysis of records of the present group of patients. The risk factors previously reported in literature were confirmed in the present study: delirious patients were slightly, but significantly older (77 vs. 74 years, $p<0.05$ ) and with a initial cognitive impairment (Inouye and Charpentier, 1996; Litaker et al., 2001; Korevaar et al., 2005). In our cohort of patients the CDT appears to be more sensitive in predicting POD than MMSE. This data implies that, when an initial cognitive derangement is present, an interruption of cortical-subcortical neuronal connections or a vascular damage with a visuo-spatial impairment and/or apraxia, as seems to be assessed by CDT (Cosentino et al., 2004), is more critical for the POD onset than a more general cognitive impairment. There is a difference in the education time, as other authors reported (Benoit et al., 2005), between delirious (9 years) and non-delirious patients (7.8 years); however, due to the variability in the small delirious group, this is not statistically significant. It is possible that with a larger number of patients this item might become significant.

The presence of depressive symptoms had a low score in both groups ( $2.5 \pm 0.2$ vs. $3.1 \pm 0.8$, ns).

Another risk factor associated with POD is the presence of functional decline in the IADL. The assessment of IADL, in our study, is more sensitive than ADL for the prediction of delirium, further indicating that an initial functional deterioration is sufficient to contribute to the onset of delirium. Nevertheless, the evaluation of functional status is an important issue in the presurgery assessment (Marcantonio et al., 1998; Litaker et al., 2001; Fukuse et al., 2005).
On one hand, the finding that a previous history of delirium increases the probability of POD is in agreement with other studies (Inouye and Charpentier, 1996; Litaker et al., 2001). On the other hand, a consumption of alcohol (Minden et al., 2005), without abuse of alcoholics, did not appear to have any influence on POD.

Finally, the evidence of significant intra-operative fall of blood pressure and hypotension, according to the indication of Marcantonio and Reich (Marcantonio et al., 1998; Reich et al., 2005) contributes to the prediction of POD. However, this event cannot be assessed prior to the surgical procedure and could only be addressed during the course of the surgery, properly constituting an intra operative risk factor.

All the hematological, biochemical or hormonal parameters were comparable between the two groups of delirious and nondelirious patients. Such observations deserve a further consideration. Usually, urological surgery is not an emergency surgery, thus the patients are well prepared for the procedure. It means that avoiding anemia, dehydration, renal failure, hormonal disorder, electrolyte changes and other confounding factors probably lowers the onset of POD, as inferred by several studies reporting the prevailing risk or precipitating factors for POD (Inouye and Charpentier, 1996; Inouye, 1998; Marcantonio et al., 1998; Litaker et al., 2001; Meagher, 2001; Cosentino et al., 2004; Fukuse et al., 2005; Korevaar et al., 2005; Minden et al., 2005; Papaioannou et al., 2005). Therefore, delirium risk is acceptably low for certain preoperative patients with few risk factors as age, functional impairment, cognitive decline, although they underwent major surgery. In our series of patients no differences were found in the onset of delirium between open or endoscopic surgery. Similarly, general or spinal anesthesia had no influence in the appearance of delirium in the postoperative period (Cohendy et al., 2005). In this study neither comorbidity nor polypharmacy, number of prescribed drugs, appear to play an important role in the POD (Table 2).

This result is in contrast with some studies reporting that opiods or psychoactive drugs with anticholinergic activity or benzodiazepines play a significant role in POD (Mendez et al., 1992; Inouye and Charpentier, 1996; Karlsson, 1999; Litaker et al., 2001; Meagher, 2001; Reich et al., 2005), as well as comorbidity (Inouye and Charpentier, 1996; Meagher, 2001). However, in the present study the opiods or psychoactive molecules were used in $<20 \%$ of the patients in both groups.

\section{Conclusions}

This study is among the first ones to address the POD incidence in elderly having urological surgery. Our findings may have some interesting implications for health care professionals involved in the preparation of this type of surgery. It is well known that an older subject with cognitive and functional decline and having a previous history of delirium shows an increased risk of POD. It should be noted that our patients are relatively healthier in comparison to other studies, which included patients with dementia or characterized by severe comorbidity ( $>4$ diseases), with polypharmacy or history of alcohol abuse; with regard to that, it could be both a limit or the strength of our study. It is evident that all these elements, which define the frail elderly phenotype, increase the probability for POD; conversely, our sample of patients may be considered as a prefrail population to which is, anyway, associated a non-negligible POD onset risk. The preoperative risk assessment for POD highlighted the relevance of cognitive and functional slight impairment, consistent with an initial cognitive deterioration. The CDT has resulted to finely predict the risk of delirium, addressing the visuospatial deficit as a more specific cognitive impairment for acute confusional state, at least in our surgical setting. This issue is interesting and quite 
innovative, deserving a further deeper insight in order to establish the validity and the appropriateness of CDT test, compared to MMSE, in the elderly preoperative risk of POD. Finally, the intra operative risk factor related to the occurrence of hypotensive episodes may be considered as an important one for the assessment of POD, whose integration with other preoperative risk factors is suggested to better stratify the profiling risk of POD. The timely treatment of significant hemodynamic events during surgery in the elderly may, in fact decrease the occurrence of POD.

The relevance of this study lies on an early preoperative multidimensional geriatric assessment, which interestingly addresses the sensitivity of CDT and IADL, respectively as cognitive and functional decline scales, in the evaluation of older patients POD risk undergoing to surgery. Further, the study focuses on the feasible integration of pre and ascertained intra operative risk factors such as hypotensive episodes to better draw the risk predictive framework for POD. A greater utilization and interpretation of this result is then supposed to improve the outcome and reduce the episodes of POD in elderly in the surgical setting.

The final take-home message is that approximately $10 \%$ of the elderly patients in this study developed POD. Age, cognitive and functional status, conduct of anesthesia and previous history of delirium are the best predictor of POD in this setting. Our findings have implications in preventing delirium in elderly by an early multidimensional evaluation focusing on the sensitivity and probable validity of CDT test and on an integrated and multidisciplinary approach to the issue.

\section{Conflict of interest statement}

None.

\section{References}

Amador, L., Goodwin, J., 2005. Postoperative delirium in the older patient. J. Am. Coll. Surg. 200, 767-773.

Benoit, A.G., Campbell, B.I., Tanner, J.R., Staley, J.D., Wallbridge, H.R., Biehl, D.R Bradley, B.D., Louridas, G., Guzman, R.P., Fromm, R.A., 2005. Risk factors and prevalence of perioperative cognitive dysfunction in abdominal aneurysm patients. J. Vasc. Surg. 42, 884-890.

Blaum, C.S., Ofstedal, M.B., Liang, J., 2002. Low cognitive performance, comorbid disease, and task specific disability: findings from a nationally representative survey. J. Gerontol. A: Biol. Sci. Med. Sci. 57, M523-M531.

Cohendy, R., Brougere, A., Cuvillon, P., 2005. Anaesthesia in the older patient. Curr. Opin. Clin. Nutr. Metab. Care 8, 17-21.

Cosentino, S., Jefferson, A., Chute, D.L., Kaplan, E., Libon, D.J., 2004. Clock drawing errors in dementia: neuropsychological and neuroanatomical considerations. Cogn. Behav. Neurol. 17, 74-84.

Fried, L.P., Tangen, C.M., Walston, J., For the Cardiovascular Health Study Collaborative Research Group, 2001. Frailty in older adults: evidence for a phenotype. J. Gerontol. A: Biol. Sci. Med. Sci. 56, M146-M156.

Fukuse, T., Satoda, N., Hijiya, K., Fujinaga, T., 2005. Importance of a comprehensive geriatric assessment in prediction of complications following thoracic surgery in elderly patients. Chest $127,886-891$.

Inouye, S.K., 1998. Delirium in hospitalized older patients: recognition and risk factors. J. Geriatr. Psychiatry Neurol. 11, 118-125.

Inouye, S.K., Charpentier, P.A., 1996. Precipitating factors for delirium in hospitalized elderly persons. Predictive model and interrelationship with baseline vulner-ability. J. Am. Med. Assoc. 275, 852-857.

Karlsson, I., 1999. Drugs that induce delirium. Dement. Geriatr. Cogn. Disord. 10, 412-415.

Korevaar, J.C., Van Munster, B.C., De Rooij, S.E., 2005. Risk factors for delirium in acutely admitted elderly patients: a prospective cohort study. BMC Geriatr. 5, 6.

Litaker, D., Locala, J., Franco, K., Bronson, D.L., Tannous, Z., 2001. Preoperative risk factors for postoperative delirium. Gen. Hosp. Psychiatry 23, 84-89.

Marcantonio, E.R., Goldman, L., Orav, E.J., Cook, E.F., Lee, T.H., 1998. The association of intraoperative factors with the development of postoperative delirium. Am. J. Med. 105, 380-384.

McCusker, J., Kakuma, R., Abrahamowicz, A., 2002. Predictors of functional decline in hospitalized elderly patients: a systematic review. J. Gerontol. A: Biol. Sci. Med. Sci. 57, M569-M577.

Meagher, D.J., 2001. Delirium: optimising management. Br. Med. J. 322, 144-149.

Mendez, M.F., Ala, T., Underwood, K.L., 1992. Development of scoring criteria for the clock drawing task in Alzheimer's disease. J. Am. Geriatr. Soc. 40, 1095-1099.

Minden, S., Carbone, L., Barsky, A., 2005. Predictors and outcomes of delirium. Gen. Hosp. Psychiatry 27, 209-214.

Papaioannou, A., Fraidakis, O., Michaloudis, D., Balalis, C., Askitopoulou, H., 2005. The impact of the type of anaesthesia on cognitive status and delirium during the first postoperative days in elderly patients. Eur. J. Anaesthesiol. 22, 492499.

Reich, D.L., Hossain, S., Krol, M., Baez, B., Patel, P., Bernstein, A., Bodian, C.A., 2005. Predictors of hypotension after induction of general anesthesia. Anesth. Analg. $101,622-628$

United Nations, 2002. Publications, ED., \& Dept. of Economics and Social Affairs, Population Division. Demographic Profile of the Older Populations: World Population Aging: 1950-2050. New York, pp. 23-27. 